ORIGINAL ARTICLE

\title{
Correlation of Fasting and Post Prandial Plasma Glucose with Hemoglobin Glycation
}

\author{
M Saiedullah $^{1} *$ S Hayat $^{2}$, SM Kamaluddin ${ }^{3}$, S Begum ${ }^{4}$ \\ ${ }^{1}$ Muhammad Saiedullah, Sr. Lecturer, Department of Applied Laboratory Sciences, Bangladesh \\ University of Health Sciences (BUHS) \& Sr. Scientific Officer, Department of Clinical Biochemistry \\ Bangladesh Institute of Health Sciences (BIHS), Dhaka, Bangladesh \\ ${ }^{2}$ Shoma Hayat, Lecturer, Department of Applied Laboratory Sciences, Bangladesh University of Health \\ Sciences (BUHS) \& Scientific Officer, Department of Clinical Biochemistry, Bangladesh Institute of \\ Health Sciences (BIHS), Dhaka, Bangladesh \\ ${ }^{3}$ Syed Muhammad Kamaluddin, Lecturer, Department of Applied Laboratory Sciences, Bangladesh \\ University of Health Sciences (BUHS) \& Sr. Scientific Officer, Department of Clinical Biochemistry \\ Bangladesh Institute of Health Sciences (BIHS), Dhaka, Bangladesh \\ ${ }^{4}$ Shahnaj Begum, Lecturer, Department of Applied Laboratory Sciences, Bangladesh University of \\ Health Sciences (BUHS) \& Sr. Scientific Officer, Department of Clinical Biochemistry, Bangladesh \\ Institute of Health Sciences (BIHS), Dhaka, Bangladesh \\ *Corresponding author
}

\section{ABSTRACT}

Association of fasting plasma glucose (FPG) and post prandial plasma glucose (PPG) on hemoglobin glycation is still controversial. In this study we aimed to assess the influence of FPG and PPG on hemoglobin glycation in newly diagnosed never treated diabetic (NDNT-DM) subjects and treated diabetic (T-DM) subjects. One hundred and seventy seven diabetic subjects were included in this study. Plasma glucose concentrations were measured by hexokinase end point technique and glycated hemoglobin $\left(\mathrm{HbA}_{1 \mathrm{c}}\right)$ levels were measured by modified cationexchange high performance liquid chromatography (HPLC). Univariate and multivariate linear regression models were applied to assess the relative contribution of FPG and PPG on HbA1c. Univariate linear regression analysis showed significant positive association of FPG and PPG with HbA1c in both groups. Multivariate regression model showed that ? (beta) value of HbA1c was $0.5528(\mathrm{p}<0.0001)$ for FPG and $0.3047(\mathrm{p}<0.01)$ for PPG in the NDNT-DM whereas $0.5509(\mathrm{p}<0.0001)$ for FPG and $0.1874(\mathrm{p}>0.05)$ for PPG in treated diabetic subjects. After adjustment for age and sex, beta remains statistically significant for FPG and PPG where beta value for FPG was higher for FPG than for PPG in both NDNT-TM group and T-DM groups. This study revealed that FPG has a stronger association on hemoglobin glycation as compared to PPG in diabetes mellitus.

Key words: Fasting plasma glucose, Post prandial plasma glucose, $\mathrm{HbA} 1 \mathrm{c}$

\section{Introduction}

Diabetes mellitus (DM) is a chronic metabolic disorder characterized by rise in blood glucose level and derangement in protein and fat metabolism ${ }^{1}$. The formation of advanced glycation end products (AGEs) plays an important role for the development and progression of the long term complications of
$\mathrm{DM}^{2}$. Glycated hemoglobin A (HbA1c) is the well characterized amadori product, produced in the early stage of AGE formation and the role of $\mathrm{HbA1c}$ in the management of DM is well established ${ }^{3-5}$. Measurement of HbA1c is an important component for the management of patients with diabetes mellitus, i.e., to monitor 
long-term glycemic status, to judge the adequacy of diabetes management and to adjust therapies ${ }^{6}$. Epidemiologic studies and clinical trial established the association of HbA1c with the risk for long term complications of hyperglycemia ${ }^{7-9}$. The A1c Derived Average Glucose (ADAG) Study defined the mathematical relationship between HbA1c and average glucose (AG), similar to the mathematical relationship between $\mathrm{HbA} 1 \mathrm{c}$ and mean plasma glucose (MPG) in the Diabetes Control and Complication Trial (DCCT) ${ }^{10,11}$. The relationship between plasma glucose levels and $\mathrm{HbAlc}$ is not consistent ${ }^{12-15}$. There are, however, insufficient data regarding the relative contribution of FPG and PPG in increasing the percentage of HbAlc. It is very desirable and also meaningful to know whether FPG or PPG, alone or in combination plays the major role for hemoglobin glycation and thereby helpful in adjusting the therapy to achieve optimal HbA1c levels. In this study, multivariate linear regression model was applied to assess the relative strength of the association between plasma glucose and HbA1c in newly diagnosed never treated diabetic subjects and treated diabetic subjects.

\section{Materials and Methods}

One hundred and seventy seven specimens were collected from newly diagnosed never treated and treated confirmed diabetic subjects during January 2012 to June 2012. Fasting and post prandial blood specimens were collected for the estimation of fasting plasma glucose, post prandial plasma glucose ( 2 hrs after $75 \mathrm{~g}$ oral glucose load and 2 hrs after breakfast) and estimation of HbA1c. Plasma glucose levels were measured by hexokinase end point techniques using Dimension RxL max automated analyzer (Siemens Healthcare Diagnostics Ltd.). $\mathrm{HbA}_{1 \mathrm{c}}$ were measured by modified highpressure liquid chromatography (HPLC) method using D-10 $0^{\mathrm{TM}}$ glycosylated hemoglobin testing system (Bio-Rad Laboratories, Inc., Hercules, CA, 94547, USA). Results are expressed as mean \pm SD. Univariate and multivariate linear regression models were applied to assess the relative strength of contribution of fasting or post prandial plasma glucose levels on hemoglobin glycation. Statistical analyses were performed by using STATISTICA version 8 for Windows.
M Saiedullah, S Hayat, SM Kamaluddin et al

\section{Results}

The mean \pm SD of age of the total study subjects was $48.4 \pm 12.3$ years. Of the total $55.9 \%$ were males and $44.1 \%$ were females. Among them $57.06 \%$ were newly diagnosed never treated diabetic subjects and $42.94 \%$ were diabetic who used anti-hyperglycemic agents for the management of DM during the last three months. Characteristics of the study subjects are presented in table I.

Table I: Characteristics of the study subjects

\begin{tabular}{lll}
\hline Parameters & $\begin{array}{l}\text { NDNT -DM } \\
(\mathrm{n}=101)\end{array}$ & $\begin{array}{l}\text { T-DM } \\
(\mathrm{n}=76)\end{array}$ \\
\hline Age (yrs) & $46 \pm 12$ & $52 \pm 11$ \\
Sex (M/F) & $59 / 42$ & $40 / 36$ \\
FBG (mmol/L) & $8.9 \pm 3.3$ & $8.8 \pm 4.1$ \\
PPG (mmol/L) & $16.0 \pm 4.3$ & $11.9 \pm 5.0$ \\
HbA 1c $(\%)$ & $9.2 \pm 2.5$ & $9.3 \pm 2.5$ \\
\hline
\end{tabular}

Univariate linear regression analyses showed that the ? (beta) value of $\mathrm{HbAlc}$ was 0.8067 $(p<0.0001)$ for FPG and $0.7654(p<0.0001)$ for PPG in the NDNT-DM group, 0.8105 $(p<0.0001)$ for FPG and $0.7388(p<0.0001)$ for PPG in the T-DM group. Multivariate linear regression analyses considering $\mathrm{HbAlc}$ as dependent variable and FPG and PPG as independent variables showed that the ? (beta) value of HbA1c was $0.5528(\mathrm{p}<0.0001)$ for FPG and $0.3047(\mathrm{p}<0.01)$ in the NDNT-DM and $0.5509(\mathrm{p}<0.0001)$ for FPG and 0.1874 $(p>0.05)$ in the T-DM group. After adjustment for age and sex, beta remains statistically significant for FPG and PPG in both NDNT-TM group and T-DM group (Table II).

Table II. Influence of FPG and PPG on HbA glycation

\begin{tabular}{lllll}
\hline Groups & \multicolumn{3}{l}{ Paramet Beta () val t-value } & $\mathrm{p}$ value \\
\hline NDNDM & FPG & 0.5548 & 5.3065 & $<0.000$ \\
& PPG & 0.3005 & 2.8672 & $<0.01$ \\
T-DM & FPG & 0.5481 & 4.7219 & $<0.000$ \\
& PPG & 0.2465 & 2.1489 & $<0.05$ \\
\hline
\end{tabular}

\section{Discussion}

In this study, we examined the relative contribution of fasting plasma glucose levels and post prandial plasma glucose levels on hemoglobin glycation in the newly diagnosed never treated diabetic subjects and treated diabetic subjects. Both univariate and multivariate linear regression analyses showed that the contribution of hemoglobin glycation is 
Correlation of Fasting and Post Prandial Plasma Glucose

higher for fasting plasma glucose levels than post prandial plasma glucose levels in both never treated and treated diabetic subjects. After adjustment for age and sex, the association between plasma glucose and $\mathrm{HbA} 1 \mathrm{c}$ remains statistically significant for FPG and PPG in both groups and contribution of fasting plasma glucose on hemoglobin glycation remains higher than the contribution of post prandial plasma glucose in both groups (Table II).

In this study, the relationship between fasting plasma glucose and $\mathrm{HbA} 1 \mathrm{c}$ is closer to $\mathrm{DCCT}^{11}$ $(\sim 0.81$ vs 0.82$)$. The correlation coefficient between fasting plasma glucose and $\mathrm{HbAlc}$ was higher than the correlation coefficient between post prandial plasma glucose and $\mathrm{HbAlc}$ in control in the study population of Masram et $\mathrm{al}^{13}(0.733$ vs 0.699$)$ whereas in type 2 diabetic subjects the relationship was found to be reversed $(0.588 \text { vs } 0.776)^{13}$. Azim et al ${ }^{14}$ also found modest relationship of FPG $(r=0.284)$ and PPG $(r=0.436)$ with HbAlc and here PPG contributed higher in the glycation of hemoglobin. Another research group found that FPG levels showed better correlations with HbA1c than with PPG levels in non-diabetic, pre-diabetic and newly diagnosed never treated diabetic subjects ${ }^{12}$. We observed similar trend of relationship between FPG and HbA1c, PPG and $\mathrm{HbAlc}_{1 \mathrm{c}}$ in both never treated and treated diabetic subjects. Hossain et $\mathrm{al}^{15}$ considered non-diabetic, newly diagnosed pre-diabetic and diabetic subjects and showed that FPG contributed higher than PPG in the glycation of hemoglobin. So the finding of this study is in concordance with the previous study done in Bangladeshi population ${ }^{12,15}$ but inconsistent with the findings of studies done in abroad. ${ }^{13,14}$ Therefore, measurement of fasting plasma glucose along with $\mathrm{HbA1c}$ may provide better outcome in the management of diabetic complications.

\section{Conclusion}

The contribution of fasting plasma glucose on hemoglobin glycation is higher than the contribution of post prandial plasma glucose levels in both newly diagnosed never treated and treated diabetic subjects.

\section{References}

1. Vinik A and Flemmer M. Diabetes and macrovascular disease. Journal of diabetes and its complications, 2002; 16: $235-45$.
2. Gugliucci A. Glycation as the glucose link to diabetic complications. J Am Osteopath Assoc 2000; 100: 621-34.

3. Jeffcoate SL. Diabetes control and complications: the role of glycated haemoglobin, 25 years on. Diabet Med 2004; 21(7): 657-65.

4. Bunn HF, Gabbay KH, Gallop PM. The glycosylation of hemoglobin: relevance to diabetes mellitus. Science 1978; 200(4337): 21-7.

5. Kasezawa N, Kiyose H, Ito K, Iwatsuka T, Kawai H, Goto $\mathrm{Y}$, et al. Criteria for screening diabetes mellitus using serum fructosamine level and fasting plasma glucose level. The Japanese Society of Multiphasic Health Testing and Services (JMHT), Fructosamine Working Committee. Methods Inf Med 1993; 32(3): 237-340.

6. Sacks DB, Bruns DE, Goldstein DE, Maclaren NK, McDonald JM, Parrott M. Guidelines and recommendations for laboratory analysis in the diagnosis and management of diabetes mellitus. Clin Chem 2002; 48: 436-72.

7. Diabetes Control and Complications Trial Research Group. The effect of intensive diabetes treatment on the development and progression of long-term complications in insulin-dependent diabetes mellitus: Diabetes Control and Complications Trial. N Eng J Med 1993; 329: 978-86.

8. DCCT Research Group. The association between glyceamic exposure and long-term diabetic complications in the Diabetes Control and Complications Trial. Diabetes 1995; 44: 968-83.

9. UK Prospective Diabetes Study (UKPDS) Group. Intensive blood-glucose control with sulphonylureas or insulin compared with conventional treatment and risk of complications in type 2 diabetes (UKPDS 33). Lancet 1988;352:837-53.

10. ADAG Study Group. Translating the hemoglobin A1c assay into estimated average glucose values. Diabetes Care 2008; 31: 1473-8.

11. Rohlfing CL, Wiedmeyer HM, Little R, England JD, Tennill A, Goldstein DE. Defining the relationship between plasma glucose and HbA1c in the Diabetes Control and Complications Trial. Diabetes Care 2002; 25: 275-8.

12. Saiedullah M, Begum S, Shermin S, Rahman MR, Khan MAH. Relationship of glycosylated hemoglobin with fasting and postprandial plasma glucose in nondiabetic, prediabetic and newly diagnosed diabetic subjects. Bangladesh Medical Journal 2011; 40(1): 37-8.

13. Masram SW, Bimanpalli. Assessment of contribution of fasting and post meal plasma glucose to increased glycated hemoglobin in diabetes mellitus - A comparative study. Int J Biol Med Res 2012; 3(3): 2020-4.

14. Azim W, Gill MM, Azim S, Farooq W. Assessment of fasting and two-hour post-prandial glucose as an economical test for monitoring of glycemic control, compared to glycated hemoglobin. Medical Channel 2011; 17(2): 5-7.

15. Hossain T, Latif ZA, Sarkar AA. Relationship of HbA1c with fasting and plasma glucose 2 hours after oral glucose load in non diabetic and newly diagnosed pre diabetic and diabetic Patients. Birdem Med J 2012; 2(2): 81-3. 